Voix et Images

voixetimages

\title{
Philippe Haeck : Les mots du vécu. Entretien
}

\section{Noël Audet et Jean Fisette}

Volume 6, numéro 3, printemps 1981

Philippe Haeck

URI : https://id.erudit.org/iderudit/200278ar

DOI : https://doi.org/10.7202/200278ar

Aller au sommaire du numéro

\section{Éditeur(s)}

Les Presses de l'Université du Québec

\section{ISSN}

0318-9201 (imprimé)

1705-933X (numérique)

Découvrir la revue

\section{Citer ce document}

Audet, N. \& Fisette, J. (1981). Philippe Haeck : Les mots du vécu. Entretien. Voix et Images, 6(3), 353-371. https://doi.org/10.7202/200278ar d'utilisation que vous pouvez consulter en ligne.

https://apropos.erudit.org/fr/usagers/politique-dutilisation/ 


\title{
Philippe Haeck: Les mots du vécu
}

\author{
Entretien avec Noël Audet et Jean Fisette
}

\begin{abstract}
«Réinventer des mythes, des symboles dans lesquels on va pouvoir s'identifier, investir, prendre plaisir"
\end{abstract}

\section{Le cheminement}

V.I. II y a eu des transformations importantes dans vos productions au fil des années soixante-dix; ce qui est un signe de dynamisme, mais aussi, peut-être, d'une sorte de malaise. Rappelons les participations aux revues, de Chroniques à Spirale, les écrits, depuis L'Action restreinte jusqu'à Naissances.

Comment, rétrospectivement, évaluer le chemin parcouru?

P.H. Eh oui! II y a eu un chemin parcouru que je puis expliquer historiquement. Mes premiers textes étaient beaucoup plus près du savoir universitaire, et je crois que c'était normal. Nattes, par exemple, était marqué par la poétique de Mallarmé; et Mallarmé, ce n'est pas un poète qu'on lit spontanément, c'est, si l'on me permet, un «poète universitaire».

V.I. Et les textes théoriques?

P.H. L'Action restreinte faisait de la linguistique et de la sémiotique une référence prédominante. Et c'était la mode, alors qu'aujourd'hui, je me suis dégagé de la linguistique; non que j'aie renié cette problématique, mais je suis passé à autre chose.

Par contre, encore aujourd'hui, comme autrefois, je crois à la nécessité de la création pour les étudiants de Lettres; puis, je suis toujours fidèle à la pensée de la modernité. Mes grandes références, mes grandes lectures, Marx, Nietzsche, Freud, demeurent constantes. Ce sont là des soucis et des intérêts toujours présents, de sorte que je puis continuer à assumer les textes qui, par ailleurs, ont quelque peu vieilli.

V.I. Il semble donc que, malgré les apparences, les positions, mêmes théoriques, n'ont pas tellement changé?

P.H. Dans Naissances, j'ai orienté mes recherches et mes intérêts vers les questions plus générales de la signification. Mais il $y$ a eu aussi les luttes qui ont changé... 
V.I. Si bien qu'aujourd'hui, il serait impossible de réécrire les articles publiés dans Chroniques?

P.H. Face à ces articles, je ne ressens aucun malaise. Je tiens encore des positions communistes. Mais je ne le fais plus, comme alors, par le biais de concepts théoriques empruntés au marxisme. J'essaie de reformuler ma pensée, de la fondre dans les mots du vécu. C'est là, je crois, la meilleure façon d'exprimer ces changements.

V.I. Cette conscience des changements ne vous amène-t-elle pas à relativiser vos prises de positions actuelles?

P.H. Il se peut qu'une certaine confiance s'estompe. Mais en fait, ce sont d'abord les enjeux, les luttes du moment qui changent. Par exemple, le discours féministe, la lutte des femmes, dont je partage généralement les revendications, atteint parfois un point au-delà duquel elle ne me nourrit plus. II faut alors, encore une fois, faire le point, relativiser, en somme: assimiler tout cela.

V.I. C'est-à-dire, atteindre, encore une fois, les mots du vécu?

P.H. Justement !

V.l. II s'agirait donc d'embrasser une cause, une lutte, en termes théoriques et de l'assimiler de sorte qu'elle devienne "connaturelle», exprimable par les mots du quotidien?

P.H. Je crois que c'est assez juste, si ce n'est que l'assimilation est en fait un travail de transformation. Et je pense que c'est là le fonctionnement normal de l'intellectuel qui est en mesure d'aller s'alimenter dans un champ théorique et, par la suite, de s'approprier ces données. C'est ainsi qu'on travaille...

V.I. Et c'est ainși qu'il n'y aurait pas de rupture brutale dans la ligne évolutive des textes?

P.H. Exactement. Au-delà des différences de surface, il y a, je crois, une grande continuité.

\section{L'enseignement}

V.I. Parlons maintenant de Naissances. On y trouvait conjoints trois lieux différents de références: l'enseignement, le combat social et l'activité de la lecture/écriture.

P.H. Je dirais que celui de la lecture/écriture se divise aussi en deux: celui qui critique, qui lit les textes des autres puis celui qui produit ses textes. Disons que là-dedans, le pôle qui m'apparaît de trop. c'est peut-être le pôle du combat social. Pour moi le pôle du combat social est à l'intérieur des trois autres; à l'intérieur d'une salle de classe, à l'intérieur d'un milieu littéraire, à l'intérieur de ma pratique d'écriture, c'est là que se 
passe l'action à chaque fois, d'une lutte sociale, d'une lutte pour changer des formes. Ainsi Naissances, c'est avant tout une lutte pédagogique, c'est là qu'est ma lutte sociale.

V.I. Vous êtes d'abord un enseignant?

P.H. Oui, et j'ai voulu m'impliquer à fond comme enseignant. J'ai eu envie de proposer une autre méthode d'enseignement. Je dirais que mes deux livres d'essais, L'action restreinte puis Naissances, inscrivent un combat social pour intervenir dans la pédagogie, dans la mesure où nos étudiants font partie de ce milieu social-là. Ils reçoivent ce que l'on appelle de la culture. J'essaye de trouver des formes d'enseignement aptes à faire des étudiants non seulement des récipients mais des gens qui vont s'essayer à créer, produire à leur tour. En réalité, l'idée peut-être centrale des essais que j'ai faits, puis de la lutte que je mène, ce serait: arrêtons de faire de l'étudiant en lettres, (pas seulement de l'étudiant en lettres mais de tous les étudiants qui suivent des cours de littérature). seulement un consommateur, forçons-le à produire aussi, pas nécessairement pour qu'il devienne écrivain, mais pour qu'il voie son travail de lecteur d'une façon différente, pour qu'il gagne en modestie, puis en même temps qu'il voie les questions d'un autre point de vue: quand on s'est essayé à plusieurs reprises à trouver son écriture, puis que ça ne vient pas, peut-être alors les questions deviennent-elles à la fois plus simples et plus difficiles: il va juger de moins haut les livres des autres, puis, peut-être avec des questions qui soient moins éloignées de la pratique de l'écrivain!

V.I. Il faut donc reposer le rapport du lecteur à l'écrivain?

P.H. J'ai trouvé que ce que j'ai pu faire dans mes premières années d'enseignement, ça ne respectait pas cet individu-là, l'écrivain qui prenait une grande partie de sa vie, prenait de grands risques, investissait beaucoup, de lui-même, alors que moi j'arrivais, puis j'appliquais mes grilles d'analyse là-dessus, en réalité en le méconnaissant presque complètement.

V.I. Il faut refuser de séparer l'engagement social des activités professionnelles? Ce qu'on reproche effectivement à une certaine conception de la littérature, c'est de poser la problématique sociale comme une queștion autonome et, ensuite, de tenter de raccrocher la littérature à cela, tant bien que mal d'ailleurs. Dans ces conditions, dire que la littérature a un rôle social, c'est projeter un désir plutôt que d'affirmer une réalité. En fait, vous dites qu'il n'y a pas d'intervention et d'engagement social possible en dehors de votre activité principale?

P.H. Oui, c'est très net pour moi, l'activité militante que je pourrais avoir au niveau social, il faut absolument que ça soit inscrit dans mon travail, celui d'enseignant, celui de critique; il faut donc que ca se passe à l'intérieur de la littérature, je me vois difficilement manifester ailleurs.

V.I. Et aussi dans le travail d'écrivain... 
P.H. Dans le travail d'écrivain aussi; c'est sûr que je cherche d'autres formes d'écriture que celles qui sont généralisées un peu partout. Si j'ai décidé d'écrire c'est justement parce qu'il n'y avait pas assez d'écritures qui me satisfaisaient; je pense même qu'on devient écrivain comme ca: on est un lecteur insatisfait puis on se dit: "Écoute donc, je vais peut-être essayer d'écrire un livre à mon goût».

V.I. Cela, c'est juste, oui. Cela nous conduit à parler du rapport entre idéologie et écriture. Entendons, par idéologie, aussi bien la révolutionnaire que l'autre. Prenons l'exemple assez fréquent chez certains de la citation. Est-ce qu'il suffit d'inscrire la citation d'un penseur révolutionnaire, Marx par exemple, pour que le poème soit révolutionnaire?

P.H. Oh! non, je ne pense pas. Moi, les citations, je les vois actuellement comme des formes de reconnaissance qu'ont les auteurs envers d'autres individus qui les ont marqués au niveau de leur pensée ou de leur écriture. Pour moi habituellement, les citations ce n'est pas tellement un salut et une référence aux critiques littéraires, aux philosophes en vogue que ce soit tel texte de Barthes, tel livre de Deleuze ou de Derrida; ça, ça m'intéresse un peu moins, ça m'agace même un peu. J'aime mieux la citation un peu inhabituelle, c'est-à-dire celle qui va aller contre la mode. Par exemple, dans mon dernier livre, il y a quatre citations au début du livre, mais je pense qu'il n'y en a aucune des quatre qui irait dans le sens de la citation qui indique l'appartenance à un clan, par exemple, celui des gens qui lisent Derrida...

V.I. Et les citations faites à l'intérieur du texte, la citation/intégration au texte?

P.H. Moi les citations/intégrations, souvent je les coule dans le texte, c'est-a-dire que je fais disparaître les guillemets, je ne les souligne pas, ou il m'arrive de faire l'inverse, c'est-à-dire de mettre des guillemets, mais ce sont de fausses citations, c'est moi qui dit le texte... mais je laisse croire au lecteur que c'est une citation, je dis atel philosophe nous dit» puis évidemment le philosophe c'est moi. Alors ca m'amuse assez. J'aime assez renverser le procédé de citation. Ailleurs, quand il y en a, c'est parce que souvent la citation telle quelle convient tout à fait à ma bouche. A ce moment-là, si je trouve telle citation, je pense à une citation de Brecht par exemple où il souhaite une bonne santé à son maître en disant: «Dors bien, mange beaucoup...», ça c'est le type de citation qui me plaît; j'aime les citations qui sont près de la vie quotidienne, pas trop de citations...

V.I. Théoriques...

P.H. Théoriques, oui c'est ça.

V.I. C'est là qu'est le problème. Est-ce qu'une citation théorique ne fait pas "naufrager» le poème, comme disait Valéry? En fait, il s'agit de la difficile intégration du théorique et de l'effet poétique.

P.H. Ma génération, celle qui a commencé à écrire avec les Herbes rouges a pratiqué à l'envie la citation théorique. Moi, au début, j'aimais ça, je 
voyais là un effet de distanciation, c'est-à-dire qu'il y a celui qui écrit et qui dit en même temps: «Regarde bien, je suis en train d'écrire, je suis en train de faire tel travail, donc ne prenez pas l'écriture pour de la vérité comme ça, immédiate».

V.I. C'est démythifier l'écriture.

P.H. Oui, sauf que je trouve que c'est devenu une mode. Puis aujourd'hui ca m'agace beaucoup. J'aime de moins en moins que quelqu'un me donne des citations théoriques à l'intérieur de ses textes poétiques. Ce qui ne m'empêche pas de poursuivre une réflexion sur l'écriture à l'intérieur de mes textes, mais j'essaye autant que possible de ne pas en faire l'élément dominant, je vise à produire des textes sur autre chose que le texte lui-même parce que ca commence à avoir été fait, puis ça a été bien fait à quelques reprises.

V.I. Il ne s'agirait plus d'afficher l'intertextualité, mais de la faire agir.

P.H. Oui, d'une certaine façon, je veux commencer à la rendre invisible quasiment par souci d'avoir plus d'effet.

V.I. Justement, on vise à rendre son texte le plus homogène possible. Et pour cela, il faut qu'il soit produit par la totalité de la personne. Dans cette perspective, la citation théorique paraît comme un corps étranger, comme une intervention extérieure consciente qui vient briser le fil de l'écriture. Et comme l'écriture relève en grande partie de l'inconscient, la citation n'est possible qu'à la condition que l'idéologie qu'elle indexe soit passée dans le vécu de l'auteur, qu'elle ait été totalement assimilée. Le plus bel exemple serait le texte de Maiakovsky où l'idéologie a été intégrée au point de passer inaperçue dans la lecture: c'est là que l'idéologie travaille le plus.

P.H. Moi, je serais tout à fait d'accord avec ça. Mais il faut bien comprendre quand même qu'on a peut-être été la première génération à avoir accès à une multitude de textes théoriques. Je pense que c'est ce qui nous a joué un tour. C'est que tout d'un coup, il y avait la linguistique, il y avait le structuralisme, la psychanalyse, il y avait la sociologie marxiste. En fin de compte, d'une génération qui avait fait des lectures qu'on peut qualifier d'impressionnistes, et qui parfois étaient valables, on s'est retrouvé, du jour au lendemain, bardé de discours théoriques. Quand on s'est mis à écrire, inévitablement, sont revenus à la surface, tous ces discours théoriques qu'on avait avalés je dirais presque en un temps record; on a raturé le retard de quatre ou cinq générations, en assimilant ces discours théoriques qui s'étaient mis à fonctionner en Europe à la fin du XIXe, début du XXe siècle.

V.I. IIs avaient été masqués...

P.H. Nous autres, c'est dans les années soixante qu'on les a lus. Devant cette profusion de discours théoriques ce fut comme si nos premiers textes, n'arrivaient pas à surnager. Prenons par exemple mon premier 
livre, Nattes. C'est évident que ce livre-là est plus marqué par une volonté calculée de produire des effets justement entre la pratique et la théorie, entre les lectures \&savantes " qu'on faisait et le vécu. C'est pour cela que ça donne une phrase si tordue et si difficile. Pour moi, en réalité, c'est le problème le plus important: comment dans la fiction, arriver à un discours, à une écriture très simple mais qui va opérer cette jonction entre la connaissance théorique que $j$ 'ai et le vécu quotidien.

\section{La lisibilité}

P.H. C'est ça que je veux réussir actuellement, trouver cette écriture presque transparente qui ferait sentir la théorie puis qui donnerait à comprendre la vie quotidienne.

V.I. C'est exactement le thème de la lisibilité.

P.H. Oui, la lisibilité me préoccupe de plus en plus; d'ailleurs je suis sorti des Herbes rouges pour cette raison. On n'acceptait pas un livre que j'avais remis, parce qu'il était devenu trop lisible.

V.I. Pas assez compliqué?

P.H. Presque cela en fin de compte, c'est-à-dire «pour qui tu te prends, tu sais très bien que la poésie c'est pour cent vingt-cinq lecteurs, alors pourquoi voudrais-tu faire un texte qui serait simple, facile».

V.I. Accessible...

P.H. Donc qui va ennuyer ces cent vingt-cinq lecteurs-là. Tu ne gagneras pas d'autres lecteurs, alors autant continuer à circuler à l'intérieur de ce groupe de cent vingt-cinq lecteurs et leur fournir les gymnastiques intellectuelles qu'ils aiment...

V.I. Qu'ils attendent...

P.H. Sauf que moi ça ne me plaisait plus ce jeu-là.

V.I. Ce souci d'agrandir le cercle des lecteurs potentiels, n'est-il pas lié à la pratique d'enseignement où on s'adresse à des gens qui sont justement hors du cercle restreint?

P.H. Oui, c'est ce qui est arrivé effectivement. J'enseigne dans un collège et les étudiants à qui je m'adresse ont dix-sept, dix-huit ans; souvent quand je leur fais lire un livre, c'est le premier qu'ils lisent, alors, nécessairement je me dis que si à dix-sept, dix-huit ans, c'est le premier livre qu'ils lisent, ca ne me sert à rien d'arriver avec tous mes concepts théoriques puis de leur plaquer ça; ils ne comprendraient rien et moi, je ne ferais rien d'autre que de leur jeter de la poudre aux yeux. Alors, il faut quasiment que je traduise toutes les théories que j'ai lues dans les mots les plus simples, avec des images tirées de la vie quotidienne pour qu'ils comprennent un peu quelque chose. Et puis là ce n'est pas 
facile dans la mesure où la théorie moderne de l'écriture a quand même renversé un paquet de préjugés qu'on avait sur le discours littéraire; mes étudiants, même s'ils n'ont pas beaucoup lu, véhiculent tous ces préjugés sur ce qu'est un texte littéraire et cela pose beaucoup de problèmes.

V.I. Est-ce que l'on pourrait mettre au compte de cette progression vers une plus grande lisibilité, cette transformation qui caractérise votre production: dans les premiers recueils, nous trouvons une versification très marquée alors que dans les derniers textes, nous sommes en face de paragraphes, de blocs très cohérents, très homogènes comme si la rupture de surface du texte avait été progressivement éliminée?

P.H. Je ne serais pas tout à fait d'accord. II n'y a de travail sur le vers que dans le premier recueil, Les Grandes lèvres. Mais je pense que ce serait plus honnête de dire que je n'ai jamais été capable de faire des vers. D'ailleurs, le vers comme moyen d'expression, ca ne me satisfait pas.

V.I. Plutôt le verset. la strophe?

P.H. Oui, d'ailleurs les poètes que j'aimais, c'était Saint-John Perse, Claudel, parce que le vers était dépassé, ça devenait un verset tellement long qu'on tombait dans la prose. Puis souvent chez les poètes, que j'aimais, que ce soit Baudelaire ou Mallarmé, c'était les poèmes en prose qui me retenaient. C'est curieux, je sens beaucoup le poème en vers comme un bibelot qui ne convient plus au monde où je suis. J'ai toujours de la difficulté à comprendre comment quelqu'un encore aujourd'hui publie un recueil de vers. Pour moi le vers c'est une forme qui est finie, qui correspondait peut-être à une forme de récitation, de rythme qui allait avec une civilisation sans doute plus lente; mais maintenant, je me dis que la vie que l'on mène à des vitesses incroyables, et aussi, une vie continuellement interrompue ne peut pas correspondre au vers qui exige tellement de lenteur. Tandis que le poème en prose me permettait une vitesse au niveau des connexions mentales, et c'est ce que j'aimais. Essayer dans un petit bloc de prose de presque tout dire d'un instant, là où cinq ou six choses se tissent entre elles.

V.I. Et le texte en prose a un quotient de représentation habituellement plus élevé que le texte en vers.

P.H. Oui, ca me plaisait aussi, la prose me permettait plus facilement d'utiliser mon vécu, ma vie quotidienne tandis qu'effectivement dans un poème en vers on dirait qu'on est limité presque au niveau des représentations possibles, c'est comme s'il y avait des choses qui ne pouvaient pas se dire dans des vers.

V.I. Mais une telle définition du petit texte en prose pourrait s'appliquer au sonnet par exemple, au poème versifié, en ce sens que c'est plein de connexions entre les différents niveaux de fonctionnement; mais ce que vous voulez dire, c'est que ça paraît un peu forcé, c'est toujours la prosodie là, qui impose une vision du monde: c'est trop restrictif. 
P.H. Oui, le sonnet c'est une contrainte très forte en fin de compte, il faut respecter les règles tandis que le poème en prose, d'une certaine façon on peut l'écrire d'un coup si on est chanceux, c'est-à-dire, si la dictée est bonne.

V.I. Serait donc prédominante dans votre activité d'écriture cette rythmique qui est une prosodie particulière au texte de prose, une prosodie sémantique?

P.H. Pour moi, la syntaxe est aussi importante: que j'aie la possibilité de déhancher une phrase très rapidement, $d^{\prime}$ une façon très abrupte tandis que les formes contraintes le permettent moins. Le sonnet, c'est plus difficile de le faire couper court et moi c'est toujours ce que j'ai senti quand j'ai essayé de faire des vers, j'avais l'impression qu'il y avait quelqu'un qui me commandait quelque chose, tandis que lorsque je fais un poème en prose, j'ai plus le sentiment d'être libre et c'est ce qui me plaît. Surtout qu'il y a toujours le problème du temps pour écrire, je n'aurais pas le temps de m'adonner avec plaisir à des formules qui exigent beaucoup de contraintes.

V.I. Est-ce que vous vous mettez plus à la place du lecteur, est-ce que vous vous relisez beaucoup?

P.H. Moi, je me lis beaucoup. J'ai une sensation assez curieuse, je lis mes livres, mes textes comme si c'était les textes d'un autre.

V.I. Vous les redécouvrez?

P.H. J'ai une très grande distance face à mes propres textes, alors je peux facilement travailler dans un texte que je me sente, comme certains auteurs, impliqué, déchiré: les textes que je fais, je l'avoue, c'est comme de petites machines, de petits moteurs.

V.I. C'est parce qu'ils sont écrits avec une distance?

P.H. Pourtant, il me semble qu'il y a des textes où on pourrait dire que je risque ma peau, je n'ai pas peur d'avancer des choses qu'on dit habituellement intimes qu'il ne faudrait peut-être pas révéler, malgré cela je ne le sens pas tellement.

V.I. C'est amusant, certains en vous lisant, constatent aussi cette distance même dans les éléments de vie intime, vous les donnez, mais en les donnant, qui sait pourquoi, il faudrait l'analyser, c'est déjà distancé, c'est déjà parlé, comme venant d'un autre.

P.H. Oui c'est ca. Effectivement je pense que le «je" qui est dans mes textes, c'est comme un «il». Quand moi j'écris, je maîtrise le «je» comme si c'était un «il».

V.I. C'est une sorte de «je» objectivé?

P.H. Je pense que ce qui arrive, c'est que, inscrivant «je», j'essaye toujours de dire du «je». 
V.I. Qu'il est comme les autres en tout cas...

P.H. Oui qu'il est comme les autres, puis en même temps, qu'il est différent; c'est assez curieux, comment j'arrive à m'en sortir ... c'est un peu comme le goût de la sécheresse...

V.I. Le goût de la sécheresse?

P.H. J'ai toujours aimé les livres secs, j'aime pas tellement les livres lyriques où il y a beaucoup de grands épanchements, c'est plutôt rare que j'aime ce genre de livre, alors qu'un livre sec, un peu raide, ça me plaît. J'ai beaucoup de goût par exemple pour la philosophie, parce que la plupart des philosophes sont monotones, ils suivent un argument d'une façon dialectique, j'aime ce type d'esprit, par exemple j'aimais beaucoup Flaubert, surtout dans Bouvard et Pécuchet; j'aime beaucoup un livre qui est toujours cassé d'une certaine façon...

V.I. Comme vous aimez la musique moderne, qui est souvent très violente?

P.H. J'aime beaucoup les musiques qu'on dit de dissonance, j'aime beaucoup la dissonance, la rupture, c'est comme si cela excitait ma curiosité, tandis qu'un grand mouvement lyrique, ma foi, j'ai l'impression qu'il faut une foi aveugle et je n'ai presque jamais ce moment de foi aveugle dans ce que je lis, dans ce que j'écris.

V.I. Oui, et c'est très cohérent: c'est-à-dire que vous écrivez comme vous lisez, ce qui est normal; mais alors n'y a-t-il pas aussi une peur d'être impliqué émotivement, d'être entraîné trop loin?

P.H. Il y a sans doute dans la distanciation une forme de protection, dans la mesure où je peux risquer dans mes textes de fiction ce qu'on appelle la vie intime, peut-être que le fait de pratiquer une écriture distancée me protège un peu. Quoique non, je ne pense pas, en fin de compte. $J$ 'ai réellement de la jouissance à pratiquer cette écriture distancée, alors que je n'avais pas de plaisir à pratiquer l'autre. C'est comme si l'autre écriture qui s'abandonne facilement, qui dit «je" volontiers... sans une certaine critique en même temps, pour moi, ça m'apparaît trop facile. Par exemple, je n'aime pas du tout les autobiographies. II faut qu'en plus du fil autobiographique il $\gamma$ ait autre chose qui travaille. Souvent dans la production contemporaine, je trouve que le fil autobiographique prend trop de place, et là je me dis pourquoi ma vie plutôt que celle d'un autre; à ce moment-là je ne vois pas l'intérêt...

V.I. On pourrait dire: pourquoi pas ma vie comme celle d'un autre. Ça dépend de ce qu'on en fait, c'est un sujet aussi intéressant.

P.H. Oui, ma vie est là dans mes livres, partout, c'est un paradoxe: il n'y a que ma vie, mais ma vie est là avec le regard critique, avec toutes sortes de préoccupations qui font de la vie un élément dans le tout; mais qu'il $\gamma$ ait dans le livre seulement ma vie, ça je n'en vois pas de bonne raison. Il faut que le tracé autobiographique de quelqu'un ait une dimension critique, poétique... 
V.I. Utilitaire, pédagogique...

P.H. Oui, presque cela.

V.I. Revenons au thème de la lisibilité et posons la question: qu'est-ce qu'il advient de l'écriture moderne dans tout cela, si l'on prend pour acquis que le projet d'écriture moderne s'est fondé sur le procès de l'idéologie de la représentation? Est-ce que vos textes devenant plus lisibles deviendraient plus représentatifs et s'éloigneraient de ce projet d'écriture moderne?

P.H. La représentation, la critique qu'on en a fait, je ne suis plus tellement d'accord avec ça. Je sais très bien que j'ai envie dans mes textes d'utiliser de la représentation, des images, des réseaux qui unissent les choses, qui reproduisent des implications et cela va s'accentuer. Je trouve regrettable les effets qu'ont entraînés toutes ces théories apprises dans les années soixante, qui étaient teintées de structuralisme, qui nous ont donc amené à faire de n'importe quoi un ensemble qui était seulement défini par des places vides en elles-mêmes de signification. Cela nous a amené à l'écriture fragmentée qui s'en va de tous bords, tous côtés, qu'on ne peut résumer, qu'on ne peut pas fixer dans des sens précis. La lecture de ces écritures-là, dans un premier mouvement, a été intéressante, dans la mesure où elle nous a appris que les anciens textes qu'on nous avait présentés comme étant doués d'unicité, de cohérence, d'harmonie, formant un tout complet, étaient en réalité fabriqués, construits. Les théories structuralistes ont émietté cela et nous ont montré les différents morceaux du tout. Où je ne suis plus d'accord, c'est quand on commence à fabriquer des livres où on ne veut plus faire un tout, où on donne seulement côte à côte tous les morceaux, puis on dit au lecteur: colle tous les morceaux puis ca va faire ton livre. Maintenant, le lecteur est intelligent, chacun va faire son propre livre. Je ne marche pas du tout: quand je vais chez l'ébéniste et que j'ai besoin d'une belle table je n'achète pas des morceaux de bois pour aller les coller chez nous, j'ai besoin d'une table achevée, finie, donc actuellement, j'ai de plus en plus besoin d'arriver à des livres qui soient des touts, qui donnent presque une idée de bien-être, où il y ait une représentation assez forte, où il y a des choses qui s'unissent, qui se lient. Je suis de plus en plus contre les formes de séparation, de fragmentation, même si j'utilise encore le fragment, je ne l'utilise pas pour séparer la réalité mais pour la reconstituer, la totaliser.

V.I. Comment retrouver dans ces propos la lecture des Herbes rouges qui est faite dans Naissances.

P.H. J'avoue que si je republiais Naissances, j'enlèverais les deux chapitres sur les Herbes rouges, mais d'un autre côté ces deux chapitres respectaient ces écritures dans la mesure où j'ai pratiqué une lecture fragmentée comme ces écritures étaient elles-mêmes fragmentées. Actuellement, je suis de moins en moins capable de lire la production des Herbes rouges, cela ne répond plus à mes attentes. Je suis tout à fait saturé de ces 
discours qui n'arrêtent pas de fuir la représentation, le sens, qui font de la vie quotidienne une scène de théâtre où placer leurs concepts ironiques, et qui en tirent une complaisance. Je souffre de cela. J'ai besoin absolument de lien, de liaison, et je me mets à relire des anciens textes dits classiques, que ce soit en philosophie ou en littérature pour me revitaliser un peu. Je trouve l'écriture formaliste, dogmatique, je pense qu'on est en train d'oublier la vie. Lorsqu'on voit les étudiants qui sont dans les universités commencer à faire des textes comme les Herbes rouges puis ne rien comprendre à ce qu'ils font, ça devient un paquet de vers vides, de phrases élégantes, qui ne nous font plus avancer.

V.I. Cette poétique obéit à une idéologie, à une vision du monde, comme l'autre, comme l'ancienne et qui est encore plus démobilisante, une fuite en avant. C'est quelque chose qui tourne à la mode, au procédé, donc à quelque chose qui n'est plus senti.

P.H. Oui, ce que l'on appelle ici l'écriture formaliste ${ }^{1}$, si elle a eu cet effet tonifiant au début pour nous montrer qu'un texte est la production, la fabrication de quelque chose, c'est devenu pour moi une préciosité, une élégance, qui ne me nourrissent plus.

V.I. Quelle lecture pouvez-vous faire alors des grands fondateurs ou des grands précurseurs de l'écriture moderne au Québec et je pense à Gauvreau, au Paul-Marie Lapointe, du Vierge incendié, au Roland Giguère des Armes blanches?

P.H. On aurait justement plus d'intérêt à relire Les Armes blanches et Le Vierge incendié plutôt que les dernières écritures formalistes dans la mesure où dans Les Armes blanches, il y avait dans chaque texte une envie de nommer la vie, un effort d'orientement comme dit Ferron, il n'y avait pas cette déroute continuelle du sens ou cette mise en boîte «savante» de la réalité, comme c'est le cas dans les textes formalistes. Quand on lit Le Vierge incendié, ce qui nous plaît, c'est cette exubérance de la sensualité, puis de la sexualité, c'était dire tout d'un coup que sensualité et sexualité existent. C'est tout le réseau de ces images-là dans Le Vierge incendié qui nous a bouleversés et qu'on a tellement aimé, provoquant un effet de signification très forte. Ce n'est plus du tout le cas maintenant, si l'on pouvait sentir la sexualité comme quelque chose de vivant puis de difficile à vivre dans Le Vierge incendié, quand les écrivains modernes parlent de la sexualité...

V.I. Ce n'est rien...

P.H. Ils en parlent comme de n'importe quoi, comme d'une machine, et pour cela les guillemets subtils et les pinces analytiques sont de rigueur, c'est devenu seulement le prétexte à une démonstration du texte qui tombe dans le vide...

V.I. Pouvons-nous résumer votre pensée de la façon suivante: ce que vous reprochez au projet formaliste des Herbes rouges, c'est que, peut-être sans trop le vouloir, on en a expulsé toute forme de sentiment? 
P.H. Oui, pourquoi pas? Voyez-vous, le mot sentiment, était un mot que je détestais il y a quelques années. Maintenant, je me dis qu'il va peut-être falloir réintroduire de tels mots. Mais au lieu de sentiment, je préférerais passion, il va falloir faire circuler de nouveau, dans les textes, de la passion et coller à cette passion d'assez près, ne pas craindre d'explorer cet univers-là, parce qu'on n'a pas le choix; si on choisit la vie, il faut absolument passer par là. Sinon, on va être des ingénieurs du texte, et on s'en fatiguera vite, car tous les textes se démontent de la même façon. Pour rendre compte des textes formalistes on pourrait presque faire le texte critique d'avance puis changer le titre et le nom de l'auteur, suivant le besoin. Cela veut dire qu'en tant que lecteur je ne lis plus rien. Je lis seulement un mode d'emploi, est-ce cela que je recherche? Non! Je vais lire un autre individu qui m'explique comment il se débrouille avec tout ce qui arrive autour de lui, qui va $\mathrm{m}^{\prime}$ apprendre à être critique, qui va m'apprendre peut-être à voir autrement ce que je vis et qui va m'empêcher de tourner en rond. Quand on lit les textes formalistes, on tourne en rond, on n'apprend plus rien, alors ça ne sert plus à rien de lire.

V.I. Cette nécessité que vous ressentez très fortement répond peut-être au besoin de violence dans le texte, comme dans la musique?

P.H. Oui, mais une violence... j'aime mieux le mot affirmation, je parlerais plutôt de puissance d'affirmation: être capable d'affirmer des valeurs fortement, même si ces valeurs-là ne sont pas à la mode, même si les milieux intellectuels peuvent les dénigrer comme étant trop simples; j'ai de plus en plus envie de risquer des choses à ce niveau-là, cela me tente beaucoup. Ce que je veux, c'est quelque chose, un sommet entre les deux, entre le versant formaliste qui tourne à vide, puis le texte trop spontanéiste qui part seulement d'impressions faciles mal comprises... je continue à dire que faire un livre, cela demande beaucoup de travail, beaucoup de patience, beaucoup de lectures, il n'est pas question d'accepter comme livres intéressants des textes qui tissent trois, quatre sentiments faciles l'un derrière l'autre mais qui ne nous en donnent pas une perception plus dense, plus intense ou plus neuve. II faut que les livres secouent quelque chose.

V.I. Ne trouvez-vous pas que la littérature, une partie de la production québécoise actuelle ne secoue plus rien précisément parce qu'elle tourne à la recette et cela ne vous gêne-t-il pas de penser que ca fonctionnait comme cela en France il y a vingt-cinq ans avec des gens comme Robbe-Grillet? Qu'au Québec, avec la poésie, on a reproduit le même fonctionnement?

P.H. Oui, c'est ça, ça a été le roman qui l'a fait en France, puis nous autres on l'a appliqué à la poésie; la génération formaliste a, ici, beaucoup aimé le nouveau roman, et quelques-uns continuent à beaucoup défendre des gens comme Robbe-Grillet et Ricardou... Je n'ai jamais aimé le nouveau roman, je n'ai jamais été un défenseur de Robbe-Grillet, Ricardou, pour la simple raison que je m'ennuyais, je n'étais pas capable de lire... j'ai toujours senti chez ces gens-là les ingénieurs du texte, 
le seul qu'on identifiait au nouveau roman et qui n'en était pas pour moi, c'était Claude Simon, lui j'arrivais à le lire, à prendre un certain plaisir, mais pour moi, Claude Simon échappe un peu à ca...

V.I. Et Beckett qui était mis là-dedans par erreur?

P.H. Beckett n'avait rien à voir avec cette école.

V.I. Est-ce que votre revendication ne serait pas d'accréditer un certain plaisir qui soit autre et plus que purement intellectuel?

P.H. Justement, je veux que le côté intellectuel soit un côté vivant, cela veut dire qu'il faut qu'il soit une partie de la vie, il faut que l'intelligence fonctionne comme le reste...

V.I. Et communique avec la sensibilité.

P.H. Bien sûr, mais c'est nous qui appauvrissons l'intelligence, car à un moment donné, elle était sensible. Ce n'était pas le cœur puis la raison, les deux étaient ensemble. II y avait cette intelligence qui pratiquait la bonté, l'accueil, qui était capable de risquer une analyse des émotions. Parmi les théoriciens modernes, c'est Barthes qui aurait dû indiquer à quelques-uns ce chemin-là, mais ce n'est pas ce qu'on a retenu de Barthes, on a retenu trop facilement le jongleur, les pirouettes les plus habiles, alors qu'il avait tenté à plusieurs reprises d'indiquer qu'il fallait revenir à un traité des passions pour lire; mais on l'a manqué, peut-être à cause d'une apparence trop formaliste des livres de Barthes.

V.I. "Formalisme», "Modernité, "Modernisme». Pourriez-vous préciser de quelles façons vous entendez et employez ces termes?

P.H. Quand je parle de formalisme, je parle d'écrivains pour qui le travail sur les formes tient lieu de travail critique, pour qui le style devient un écran une fin, ils, elles adoptent la posture artiste, j’y vois une espèce de fuite; ce que j'oppose à une telle posture ce sont les écrivains pour qui l'écriture n'est qu'un moyen d'y voir plus clair dans leur vie et par le fait même dans la vie au jour le jour des femmes, des hommes, des enfants avec qui ils, elles vivent. On peut mettre cette opposition en parallèle avec les autres notions: les formalistes seraient du côté du modernisme, de la mode, souvent arrogante comme le note Lefebvre dans son Introduction à la modernité, tandis que les autres plus branchés sur l'histoire que sur l'art seraient du côté de la modernité, de la critique. La modernité. c'est la pensée critique telle qu'elle a commencé à être élaborée à la fin du XIXe siècle: en sociologie on pense à Marx, en philosophie on pense à Nietzche, en psychologie on pense à Freud, ce sont les trois grandes têtes qui restent des lectures essentielles. Quand on les lit, on découvre des textes en mouvement qui étaient toujours très près de la réalité de leur époque, il n'y avait rien de formaliste, il n'y avait pas de recette là-dedans... Ceux que j'appelle modernistes, ce sont justement de mauvais disciples de ceux qui ont créé cette pensée critique extrêmement vivante... 
V.I. Et qui l'ont sclérosée...

P.H. Qui l'ont sclérosée, qui ont pris des vérités qui étaient en train de se faire, en mouvement, qui ont pris même parfois des hypothèses et qui en ont fait des dogmes.

V.I. Pire que les avoir sclérosées ils en ont fait une orthodoxie...

P.H. C'est ce qui paralyse actuellement la création.

V.I. On doit constater que depuis quelques années vous avez changé suivant un rythme accéléré; ce sont vraisemblablement les ruptures dont il était question tout à l'heure?

P.H. Oui, et pour arriver là, pour arriver à affirmer mes valeurs, il a fallu que je quitte les groupes où il y avait un discours de censure, ce qui importe pour moi c'est quelqu'un qui encourage la production, la création, qui n'a pas tendance à tout fixer, immobiliser; et comment ne pas remarquer que les gens dits de gauche agissent souvent comme les gens de droite, ils paralysent tout; où est la gauche, où est la droite? De toute façon, je n'ai plus besoin d'être à droite, d'être à gauche, j'ai de plus en plus envie de travailler comme moi je l'entends, en collant le plus à mes envies et à mes désirs, je commence à être fatigué de me faire donner des leçons sur ce qu'il faut faire pour faire une écriture moderne.

V.I. Et alors le travail de l'intellectuel, c'est précisément d'être libre à tout point de vue, c'est-à-dire de pouvoir concevoir, articuler une pensée sociale en tenant compte du réel vécu?

\section{Les racines}

P.H. Je pense que l'intellectuel devrait être celui qui apprend à jouir. Il faut que l'intellectuel cesse d'être uniquement celui qui analyse, il faut qu'il devienne créateur; il me semble qu'il n'y a que les créateurs qui peuvent apprendre à jouir.

V.I. Oui et à réinventer la vie en fonction de ça...

P.H. II faut que la pensée soit jouissance, on n'est pas là pour censurer tout le monde, puis se censurer par le fait même, s'interdire des choses qui nous feraient plaisir. Je pense à une chose: quand on lisait, on s'interdisait toute identification. II fallait lire scientifiquement, c'est bien dommage. Quand je lis maintenant, je renoue avec cette ancienne loi-là qui fait que dans un livre, il faut qu'il $y$ ait quelque chose avec lequel je m'identifie, pour que je travaille à partir de ce point-là.

V.I. Mais pas nécessairement un personnage? Ce n'est pas nécessairement une identification psychologique?

P.H. Pas nécessairement un personnage, mais ça peut être un personnage, ca se pourrait très bien qu'en lisant un roman je m'identifie à une femme, 
que j'aie plaisir à cette identification-là, pourquoi pas? Pourquoi me l'interdire à cause de leçons théoriques qui arriveraient de quelque part?

V.I. Comment lisez-vous Victor-Lévy Beaulieu ou Gilbert Larocque?

P.H. Victor-Lévy Beaulieu, je l'aime beaucoup pour une raison qui m'est très personnelle: assez curieusement, des fois j'ai l'impression que j'ai la même vie que lui; je me l'explique mal parce que lorsque je regarde les faits l'un à côté de l'autre cela n'a pas l'air de marcher, mais pourtant on dirait de certaines expressions, un certain ton, que ce sont les miens tels quels, entre autres, tout le lien qu'il y a entre les fils et les pères. je suis très sensible, dans les textes de Victor-Lévy Beaulieu, à ce qui se passe entre le fils et le père : c'est comme s'il y avait un grand appétit de tendresse d'un côté puis de l'autre qui ne se réalise jamais. C'est-àdire que le fils manque le père, puis le père manque le fils, alors que leur désir le plus grand serait de se rejoindre. Dans ma biographie, c'est ce que je vois, j'ai eu plusieurs pères, que j'ai admirés, que j'ai été ensuite amené à décapiter. J'en suis à une étape où j'aimerais qu'il se passe quelque chose entre tous ces pères que j'ai eus puis que j'ai décapités. Seulement c'est très difficile, c'est comme s'il fallait que j'invente quelque chose, une relation nouvelle entre le fils et le père, que je n'y arrivais pas. Quand je lis Victor-Lévy Beaulieu je ressens à chaque fois cette grande nostalgie.

V.I. Parce que lui a réussi?

P.H. Non, c'est pour cela que je dis que je me sens près de lui, que je m'identifie tellement à ses livres, cet immense appétit, cette immense soif que nos pères nous parlent, puis quand ils nous parlent, on n'entend pas; et, à l'inverse, les fils se mettent à parler, puis les pères ne sont pas plus capables d'entendre.

V.I. Plus tôt, il était question de lecture d'identification, vous nous en donnez un bel exemple ici, il serait peut-être plus juste de parler d'une lecture impliquant un investissement émotif.

P.H. Oui, ce serait peut-être plus juste. Avec Victor-Lévy Beaulieu pour moi l'investissement est très fort à ce niveau-là, et pourtant dans ses livres ce n'est souvent que quelques passages; dans Don quichotte de la démanche, que je trouve un roman extraordinaire, le père apparaît seulement deux ou trois fois mais à chaque fois je sais ce qui se passe... Je dirais que c'est ça qui est partout, même quand il parle d'autres choses.

V.I. Et le rapport à la mère? Est-ce vrai qu'il a toujours été occulté dans la production québécoise?

P.H. Oui, c'est assez vrai. J'ai toujours le projet de faire des textes autour de ma mère. Le rapport à la mère je pense que c'est un des rapports les plus difficiles dans la mesure où justement, j'ai eu une biographie complètement dominée par des images de pères, les femmes étaient absentes, les femmes n'avaient pas de voix sinon sous forme de rires 
et de larmes, il n'y avait pas de discours qui pouvait être tenu par les femmes, donc l'identification s'est toujours passée avec les pères; et peut-être à cause de cette grande vague de textes féminins, je commence à voir comment tous ces pères que j'ai admirés ont complètement enfoncé la voix des femmes, les voix de leurs femmes, les voix de leurs filles; d'ailleurs ca me fait souffrir encore beaucoup, ca me fera toujours beaucoup souffrir que ma mère ne puisse pas lire ce que j'écris. Et sans doute ne serais-je pas plus capable d'entendre ma mère. Dans Victor-Lévy Beaulieu il y a la même chose, ça ne se fait pas plus entre le fils et la mère. J'aime de plus en plus ma mère, j'essaye de trouver un langage qui la rejoindrait, lui serait audible, je commence à avoir plaisir aux choses simples qu'elle me dit, alors qu'avant c'était des choses qui me paraissaient insignifiantes, que je méprisais; notre identification très forte au père, nous a fait manquer presque toutes les valeurs sensuelles du vécu. Ça nous a beaucoup aidé à être des intellectuels très habiles mais on a perdu cette ressource, cette vie. Puis moi, je répèterais les grands lieux communs qui disent que la femme est peut-être plus près des éléments naturels, plus près de la terre, la femme est plus terrienne, moins limitée à son cerveau que ne peuvent l'être les hommes. Je me suis trouvé tout d'un coup avec un immense appétit pour développer autre chose que le cerveau, je me suis mis à lire beaucoup de textes de femmes, j'ai vu comment moi, par mon discours, j'écrasais ma femme, comment j'avais toujours écrasé ma mère, comment mes pères avaient fait la même chose. J'ai vu toute cette chaîne infinie, le discours d'un sexe qui domine l'autre. Alors pour moi l'écriture féminine a été une révolution, puis ça continue à l'être. C'est peut-être le fait le plus important de l'activité littéraire au XXe siècle que cette montée de l'écriture féminine; puis cette écriture a aussi eu le mérite de révéler a chaque homme sa partie féminine. S'il n'y avait pas eu toutes ces écritures féminines, peut-être que j'aurais toujours continué à censurer dans mon discours, dans mon écriture, ce qu'on peut appeler la part lyrique ou la part inconsciente, celle qu'on peut maîtriser le moins, celle qui vient comme des débordements d'images, celle qu'on serait parfois assez mal pris d'exprimer rationnellement. Donc, cette part féminine-là, pour moi, c'est ma quête. J'essaie de donner de la place à la féminité dans mon esprit, dans mon écriture. De ce point de vue-là, j'essaie de faire attention aux discours des femmes qui m'entourent à commencer par celui de ma mère bien sûr, de ma femme, mais ce n'est pas facile. En même temps, il se produit un autre phénomène: je me sens mieux avec les femmes parce qu'elles m'ouvent un domaine inconnu que j'avais envie de connaître, un domaine qui multiplie nos possibilités au niveau de la connaissance; d'un autre côté, je suis devenu aussi plus, je dirais, tendre pour les hommes, après avoir passé une période peut-être auto-punitive: j'en arrivais à avoir honte d'appartenir à cette lignée dominante qui a écrasé des milliers de femmes pendant des siècles.

\section{V.I. La culpabilité?}


P.H. Oui, c'est ça, dire: «je ne parle plus à des salauds comme ça puis je ne me parle plus. Je me mets à parler comme une femme, puis je renie presque mes discours d'homme». Maintenant, c'est fini, je n'ai plus du tout le goût de lancer à la lignée à laquelle j'appartiens des paquets de briques, et de tout démolir. Je trouve que, à sa façon, la lignée masculine n'a pas été plus heureuse que la lignée féminine quand je regarde toute cette lignée masculine forcée à des performances, forcée à remporter des succès, à prendre des positions de chef...

V.I. A jouer des rôles...

P.H. Oui, à jouer des rôles, il n'y a rien de tellement enviable là-dedans. Cela nous a privé d'un tas de plaisirs sensuels, manque de contact au niveau du corps avec les enfants, avec les êtres humains en général. Quand je regarde tout ceux qui ont été mes pères, je ne suis plus prêt à les décapiter, j'essaye plutôt de voir par quelles histoires ils ont passé, par quelle histoire moi je continue de passer, puis tranquillement, j'essaye de bouger un peu, mais je sais bien que j'en ai des pères dans la peau, par hérédité; c'est sûr que je n'arriverai jamais, sans doute, au comportement qu'une femme pourrait rêver, pourrait attendre. Mais je pense que certaines femmes commencent à le comprendre, commencent à voir que les hommes aussi ont eu leur formation très contraignante, à s'apercevoir qu'en fin de compte, souvent la position dominante n'est pas si intéressante que ça. La position dominée chez les femmes les a mises en contact avec ce que j'appelle les éléments naturels où la vie était plus facile à sentir, une vie où l'on était moins compulsif, moins pressé. Il y a peut-être eu malgré tout chez les femmes une plus grande jouissance de la vie que chez les hommes qui ont peut-être beaucoup joui du pouvoir mais on s'aperçoit qu'entre jouir du pouvoir et jouir de la vie, le choix commence à être facile, on a de plus en plus envie de jouir de la vie.

\section{V.I. Et Dieu?}

P.H. J'ai été un croyant, un pratiquant; c'était très fort; jusqu'à vingt-cinq ans la prière pour moi a été une activité très dynamique; ça a été très important, c'était une des réserves que j'avais pour développer de la force, de la puissance. Par après, m'instruisant, fréquentant les universités, découvrant toutes ces pensées qu'on a dit de la modernité, j'ai bien vu comment les institutions que gérait l'Église avaient entretenu souvent la majorité de leurs croyants dans l'ignorance dans certains secteurs, en particulier dans les sciences, dans la sexualité. Je trouvais ca épouvantable. J'ai eu une réaction assez hostile contre les prêtres, les curés; maintenant, ca aussi c'est calmé; je serais même porté à croire aujourd'hui que les formes religieuses ont quelque chose de valable dans la mesure où elles sont vivantes, c'est-à-dire que les images, les symboles qui sont véhiculés par ces formes religieuses permettent réellement un échange entre les croyants, entre ceux qui adhèrent à cet ensemble de symboles et d'images; et si ces images, ces symboles développent plus de générosité, moi j'encouragerais le développement de ces choses- 
là, mais je continue à être tout à fait hostile à un discours religieux de type sacrificiel ou punitif. Des formes de religion qui sont des formes religieuses, des formes qui invitent au partage, c'est mon idéal communiste, que ça prenne le détour de symboles religieux au lieu de symboles politiques cela ne me dérange pas dans la mesure où ça peut créer des êtres qui aient cette qualité de ne pas se sentir surveillés, qui n'aient pas peur de se faire voler une place, qui ne se laissent pas prendre au jeu de la concurrence. De ce point de vue, je regretterais qu'on ait perdu la possibilité de générosité qui était inscrite dans les formes religieuses et qu'on ait pas su les remplacer au niveau politique par exemple. Le problème le plus important actuellement c'est toutes ces formes qu'on a abattues, détruites qui étaient souvent des formes d'unification, de liaison; en les déconstruisant on n'a pas voulu se donner d'autres formes symboliques, d'autres formes qui nous permettent de nous lier ensemble. C'est ça qui est très grave et je crois que c'est précisément cette nonappartenance à des communautés symboliques, c'est-à-dire des collectivités qui ont des valeurs communes fortes, qui explique les recours aux psychotérapies et aux analyses. Avec notre intelligence très vive on a systématiquement coupé tous les liens: tu tues les pères, tu tues les formes religieuses, tu tues les formes sentimentales, tu tues un ensemble de formes qui n'avaient pas d'autre fonction que d'assurer une circulation des échanges.

V.I. C'est la fonction symbolique qui était remise en cause, c'est-à-dire toute la symbolique de la vie.

P.H. C'est ca, pour moi, ce qu'on a tué, c'est le symbole, ce qui ré-unit.

V.I. Le fonctionnement symbolique.

P.H. On a tué la fonction symbolique. Puis notre tâche maintenant au niveau littéraire, c'est de la réinventer. II va falloir réinventer des mythes, des images, des symboles dans lesquels on va pouvoir s'identifier, investir, prendre plaisir, on est pris avec ça, c'est une tâche immense.

V.I. Terminons maintenant sur l'œuvre à faire, à venir. Qu'est-ce qui se prépare?

P.H. II y a le livre qui s'en vient, qui s'appelle La Parole verte, juste le titre est déjà un indice: pour moi, La Parole verte, c'est la parole franche, c'est la parole simple qui ne prend pas de détours, qui y va directement au risque parfois même d'être presque simpliste dans sa formulation, mais ça ne me dérange pas. Je me dis qu'il faut faire quelques livres comme ca qui vont presque choquer à cause de leur apparente banalité; il faut se donner une famille de livres comme ça qui créent une réserve, et qui, un jour, produisent peut-être de meilleurs livres encore. Là, on est pris à les inventer ces livres-là. Quand je regarde la production québecoise, je cherche ce que j'appelle des livres de santé, des livres que je lirais puis qui me feraient sentir bien dans ma peau, qui me donneraient à jouir toutes sortes de petites choses. Et ces livres-lá sont toujours rares, il y 
en a eu quelques-uns, ces deux dernières années, je pense à "La Marche» de France Théoret dans son dernier livre Nécessairement putain, que je viens tout juste de lire, à la générosité de Charron dans Peinture automatiste, au roman de Madeleine Gagnon Lueur, au Monsieur Melville de V.-L. Beaulieu que j'ai lu à moitié je me garde l'autre moitié, à l'essai de Chamberland Terre souveraine, aux deux premières nouvelles de Noël Audet dans Quand la voile faseille, au dernier roman de Madeleine Ouellette-Michalska, Le Plat de Lentilles². J'ai bien aimé ce dernier roman, Madeleine Michalska installe une femme qui s'éveille, qui prend conscience, alors évidemment c'est intéressant; moi j'aimerais qu'il y ait cela pour les hommes, c'est-à-dire que tout d'un coup, on pense à des hommes qui soient heureux, qui trouvent l'air bon à manger comme dit Jean-Jules Richard.

V.I. Ce que vous aimeriez produire?

P.H. Mon rêve, ce serait d'écrire un ou deux romans comme j'aimerais les lire, à savoir placer ces hommes dans Montréal en 1980, avec la vie moderne; et que, tout d'un coup, ces hommes-là ne soient plus des hommes minés par le désespoir, le cynisme, mais des hommes qui soient capables de s'ouvrir à autre chose, d'entendre les rumeurs de la vie. Je sais que cela doit être difficile. Comment imaginer des livres comme cela alors qu'on est pris d'une part soit avec des livres très intellectuels, très analytiques, très formalistes, ou d'un autre côté avec des livres qui n'en sont pas, qui se contentent de faire des pirouettes ou de remplir des pages avec des recettes qui sentent le moisi depuis longtemps. J'ai envie d'une écriture presque de simple transparence, j'ai même envie de romans avec des personnages, des situations, surtout pas des romans avec des pronoms; j'aurais envie de voir du monde vivant dans les livres, du monde vif, du monde auquel tu peux adhérer, que tu aurais, comme autrefois, envie de rencontrer. Quand on lisait un ancien roman, on se disait : j'aurais aimé une femme comme ça ou un homme comme ça. On aurait besoin de romans comme ça, qui nous disent d'être ces hommes, ces femmes dans notre vie.

Entrevue réalisée le 4 octobre 1980

par Noël Audet et Jean Fisette

1. Pour indiquer ce que j'entends par écriture formaliste, je renvoie au corpus suivant: les travaux de Nicole Brossard, Roger Des Roches, André Roy, Claude Beausoleil, Louis-Philippe Hébert, Normand de Bellefeuille, Marcel Labine, Hugues Corriveau.

2. II $y$ a aussi des livres que j'aime même s'ils produisent en moi un certain malaise je pense par exemple à Blessures de François Charron, à Pylônes de Joseph-Henri Le Tourneux, à la Promeneuse et loiseau de Denise Desautels, a De la contradiction de deux étreintes de François Martel, à L'implicite/le filigrane de Michel Gay, à Des voix la même de Louise Bouchard. 